\title{
Quantifying Structures in the Ocular Fundus
}

\author{
Douglas R. Anderson ${ }^{1}$ and Claude Burgoyne ${ }^{2}$
}

From the ${ }^{1}$ Bascom Palmer Eye Institute, University of Miami Miller School of Medicine, Miami, Florida;

danderson@med.miami.edu; and ${ }^{2}$ Discoveries in Sight Research Institute, Devers Eye Institute, Portland, Oregon.

Moghimi et al. showed that Heidelberg retinal tomography (HRT) and one brand of spectral domain optical coherence tomography (SD-OCT) do not give the same measurement of disc and neuro-retinal rim areas within the disc (http://dx.doi.org/10.1167/iovs.118362). ${ }^{1}$ The measurements correspond better with an attempt to correct for optical magnification (by taking into account axial length of the eye). However, the definition of "the edge of the disc," which enters into calculations of the disc area and rim area, is not the same for these two instruments. It often is a boundary defined as the termination of Bruch's membrane for OCT, but by other landmarks for the HRT or clinical examination. ${ }^{2,3}$

As further work is done comparing new imaging instruments, and attempts are made to standardize the measurements, other differences in methods will be relevant, such as differences in nature of the signal, image processing, segmentation of layers, reference planes, and the geometric strategy for rim width measurement. In this regard, several groups (most recently Reiss et al. ${ }^{3}$ ) proposed that rim width might be calculated advantageously in a "minimum" fashion (perpendicular to the trajectory of the axons) from the end of Bruch's membrane to the cup surface obliquely, instead of measured "horizontally" within different measurement planes that often are related to the termination of Bruch's membrane for OCT, but variably located for HRT. Making comparison more difficult is that any component of the rim measurement that occurs in the horizontal direction is influenced by the transverse magnification mentioned previously, but thickness measurements in the axial direction are not. Moreover, thickness of some retinal structures change with location, so that measurements depend on consistently positioning the array of a-scans. ${ }^{4}$

The practical import is that the measurements from these new diagnostic machines are not interchangeable. Even two different OCT models don't give identical values. ${ }^{4}$ Further studies will add to our understanding of the sources for discrepancies, and perhaps suggest means to make measurements readily comparable.

\section{References}

1. Moghimi S, Hosseini H, Riddle J, et al. Measurement of optic disc size and rim area with spectral-domain OCT and scanning laser ophthalmoscopy. Invest Ophthalmol Vis Sci. 2012;53:4519-4530.

2. Sharma A, Oakley JD, Schiffman JC, Budenz DL, Anderson DR. Comparison of automated analysis of Cirrus HD-OCT spectral domain optical coherence tomography with stereo photographs of the optic disc. Ophthalmology. 2011;118:1348-1357.

3. Reis AS, O'Leary N, Yang H, et al. Influence of clinically invisible, but optical coherence tomography detected, optic disc margin anatomy on neuroretinal rim evaluation. Invest Ophthalmol Vis Sci. 2012;53:1852-1860.

4. Patel NB, Wheat JL, Rodriguez A, Tran V, Harwerth RS. Agreement between retinal nerve fiber layer measures from Spectralis and Cirrus spectral domain OCT. Optom Vis Sci. 2012;89:E652-E666. 